\title{
Key Role of Sulfonylurea Receptor 1 in Progressive Secondary Hemorrhage after Brain Contusion
}

\author{
J. Marc Simard, ${ }^{1,2,3}$ Michael Kilbourne, ${ }^{5}$ Orest Tsymbalyuk, Cigdem Tosun,, John Caridi, \\ Svetlana Ivanova, Kaspar Keledjian,, Grant Bochicchio,,6 and Volodymyr Gerzanich ${ }^{1}$
}

\begin{abstract}
An important but poorly understood feature of traumatic brain injury (TBI) is the clinically serious problem of spatiotemporal progression ("blossoming") of a hemorrhagic contusion, a phenomenon we term progressive secondary hemorrhage (PSH). Molecular mechanisms of PSH are unknown and efforts to reduce it by promoting coagulation have met with equivocal results. We hypothesized that PSH might be due to upregulation and activation of sulfonylurea receptor 1 (SUR1)-regulated $\mathrm{NC}_{\mathrm{Ca} \text {-ATP }}$ channels in capillary endothelial cells, predisposing to oncotic death of endothelial cells and catastrophic failure of capillary integrity. Anesthetized adult male rats underwent left parietal craniectomy for induction of a focal cortical contusion. The regulatory subunit of the channel, SUR1, was prominently upregulated in capillaries of penumbral tissues surrounding the contusion. In untreated rats, PSH was characterized by progressive enlargement of the contusion deep into the site of cortical impact, including corpus callosum, hippocampus, and thalamus, by progressive accumulation of extravasated blood, with a doubling of the volume during the first $12 \mathrm{~h}$ after injury, and by capillary fragmentation in penumbral tissues. Block of SUR1 using low-dose (non-hypoglycemogenic) glibenclamide largely eliminated PSH and capillary fragmentation, and was associated with a significant reduction in the size of the necrotic lesion and in preservation of neurobehavioral function. Antisense oligodeoxynucleotide against SUR1, administered after injury, reduced both SUR1 expression and PSH, consistent with a requirement for transcriptional upregulation of SUR1. Our findings provide novel insights into molecular mechanisms responsible for PSH associated with hemorrhagic contusions, and point to SUR1 as a potential therapeutic target in TBI.
\end{abstract}

Key words: contusion; glibenclamide; hemorrhage; sulfonylurea receptor 1; traumatic brain injury

\section{Introduction}

$\mathbf{H}$ UMAN TRAUMATIC BRAIN INJURY (TBI) is a complex multifaceted disease. Many of the different features of TBI experienced by humans have been studied in various animal models, including clinical abnormalities such as vestibulomotor and cognitive dysfunction, epilepsy, coma, and death, as well as pathophysiological abnormalities such as ischemia, edema, excitotoxicity, and diffuse axonal injury (Buki and Povlishock, 2006; Marmarou, 2007; Morales et al., 2005).

Arguably, one of the most important, yet least studied, aspects of TBI is the serious problem of spatiotemporal progression ("blossoming") of a hemorrhagic contusion. We refer to this phenomenon as progressive secondary hemorrhage (PSH), to emphasize its secondary nature and hence its potential treatability. Between $25 \%$ and $50 \%$ of traumatic hemorrhagic contusions and hematomas show radiographic evidence of progression on serially obtained head computed tomographic (CT) scans during the acute post-injury period (Chang et al., 2006; Oertel et al., 2002; Servadei et al., 1995; Smith et al., 2007). Hemorrhagic expansion incites further injury to the surrounding brain, due not only to local mass effect, but also to biochemical toxicity induced by various pathophysiological cascades. Although coagulopathy may contribute to PSH, treatments aimed at correcting clinical or sub-clinical bleeding disorders have shown only modest benefit (Narayan et al., 2008), suggesting possible involvement of mechanisms unrelated to coagulation.

The sulfonylurea receptor 1 (SUR1)-regulated $\mathrm{NC}_{\mathrm{Ca}-\mathrm{ATP}}$ channel is a $30-p S$ channel that conducts monovalent but not divalent cations (Simard et al., 2008). This channel is not

Departments of ${ }^{1}$ Neurosurgery, ${ }^{2}$ Pathology, ${ }^{3}$ Physiology, and ${ }^{4}$ Surgery, University of Maryland School of Medicine, Baltimore, Maryland. ${ }^{5}$ Department of Surgery, Walter Reed Army Medical Center, Washington, D.C.

${ }^{6} \mathrm{R}$. Adams Cowley Shock Trauma Center, Baltimore, Maryland. 
constitutively expressed, but is newly upregulated in neurons, astrocytes, and capillary endothelial cells after central nervous system (CNS) ischemia or trauma. The channel is inactive when expressed, but becomes activated when intracellular ATP is depleted, with activation leading to cell depolarization, cytotoxic edema, and oncotic cell death (Chen et al., 2001; Chen et al., 2003; Simard et al., 2006). Pathological involvement of the channel in capillaries results in formation of ionic and vasogenic edema (Simard et al., 2006; Simard et al., 2009b; Simard et al., 2009a), has been postulated to predispose to hemorrhagic transformation in the context of ischemia (Simard et al., 2007a), and has been shown to be responsible for secondary hemorrhage following spinal cord trauma (Simard et al., 2007b).

We hypothesized that SUR1-regulated $\mathrm{NC}_{\mathrm{Ca}-\mathrm{ATP}}$ channels might be involved in PSH following brain contusion. Here we used a rat model of focal cortical contusion to evaluate this hypothesis. In brief, we found that SUR1 was prominently upregulated in penumbral capillaries in the region of contusion, and that inhibition of SUR1 by glibenclamide or by antisense directed against SUR1 largely eliminated the PSH characteristic of contusion, resulting in a significant reduction in lesion size and preservation of neurobehavioral function. Our findings provide novel insights into the molecular mechanisms responsible for blossoming of hemorrhagic contusions, and point to SUR1 as a potential therapeutic target in TBI.

\section{Methods}

\section{Focal cortical contusion}

All surgical procedures were approved by the Institutional Animal Care and Use Committee of the University of Maryland. An investigator who was blinded to the treatment performed all of the surgical procedures. Male Long-Evans rats (275-300 g; Harlan Laboratories, Indianapolis, IN) were anesthetized $(60 \mathrm{mg} / \mathrm{kg}$ ketamine and $7.5 \mathrm{mg} / \mathrm{kg}$ xylazine IP) and allowed to breathe room air. The head was fixed in a stereotaxic frame and an 8-mm craniectomy positioned 1-2 $\mathrm{mm}$ from the sagittal and lambdoidal sutures was performed in the left parietal region, taking care to avoid injury to the dura and underlying cerebral cortex. Body temperature was maintained at $37^{\circ} \mathrm{C}$ using a heating pad (Deltaphase Isothermal Pad; Braintree Scientific Inc., Braintree MA). Arterial blood gases, hematocrit, electrolytes, and glucose (i-STAT; Heska Corp., Fort Collins, CO) immediately prior to injury were (mean $\pm S D$ ): partial oxygen pressure $79 \pm 9 \mathrm{~mm}$ $\mathrm{Hg}$; oxygen saturation $94 \pm 2 \%$; partial carbon dioxide pressure $42 \pm 10 \mathrm{~mm} \mathrm{Hg}$; total carbon dioxide $24 \pm 5 \mathrm{mmol} / \mathrm{L}$; $\mathrm{HCO}_{3}, 23 \pm 5 \mathrm{mmol} / \mathrm{L}$; base excess, $-2.5 \pm 5 \mathrm{mmol} / \mathrm{L} ; \mathrm{pH}$ $7.3 \pm 0.0$; hematocrit $33 \pm 6 \%$; hemoglobin $11.3 \pm 2.0 \mathrm{~g} / \mathrm{dL}$; $\mathrm{Na}^{+} 144 \pm 6 \mathrm{mmol} / \mathrm{L} ; \mathrm{K}^{+} 3.4 \pm 0.8 \mathrm{mmol} / \mathrm{L}$; ionized calcium $1.3 \pm 0.2 \mathrm{mmol} / \mathrm{L}$; and glucose $288 \pm 62 \mathrm{mg} / \mathrm{dL}$. Focal cortical contusion was induced using a modified Feeney device (Feeney et al., 1981) in which their 4.5-mm flat impactor (footplate) was replaced with a $4.8 \mathrm{~mm}$ Teflon sphere, and the depth of depression was not limited. The vertical guide tube (inside diameter $6 \mathrm{~mm}$ ) and the spherical impactor were gently positioned on the dura inside the craniectomy. The impactor was activated by dropping a $10 \mathrm{~g}$ weight $5 \mathrm{~cm}$ down the shaft of the guide tube onto the Teflon sphere (impact velocity $1 \mathrm{~m} / \mathrm{sec}$ ). In all cases the weight was removed within $\sim 3$ sec of impact. The dura was never lacerated by this procedure. After injury the craniectomy was left open and the scalp was closed. The rats were recovered and observed for various times up to 7 days, after which they were euthanized using a lethal dose of sodium pentobarbital IP.

\section{Experimental series}

For the studies reported here, 119 rats underwent the contusion injury described above. Of these, 2 were excluded for early death due to anesthesia, and 5 were excluded for subdural hematoma. The remainder comprised four series, as follows. In series 1 (23 rats), 9 rats were used for hemorrhage, 5 for immunohistochemistry, 3 for in-situ hybridization, and 6 for immunoblot. In series 2 (49 rats), 34 were used for hemorrhage at five different times (vehicle versus glibenclamide; Veh vs. GLIB), 9 for hemorrhage at $24 \mathrm{~h}$ (Abcc8-Scr versus Abcc8-AS), and 6 for immunoblots at $24 \mathrm{~h}$. In series 3 (26 rats), 6 were used for immunohistochemistry (Veh vs. GLIB), and 20 were used for histology (Veh vs. GLIB), all at $24 \mathrm{~h}$. In series 4 (14 rats), all were used for neurobehavioral testing during 1 week (Veh vs. GLIB).

\section{Block of sulfonylurea receptor 1}

Within $10 \mathrm{~min}$ of contusion injury, a loading dose of glibenclamide was given $(10 \mu \mathrm{g} / \mathrm{kg} \mathrm{IP})$, and a mini-osmotic pump (1.0 $\mu \mathrm{L} / \mathrm{h}$ with an Alzet 2001; Durect Corporation, Cupertino, CA) was implanted for continuous infusion of drug subcutaneously. Controls received the same volume of vehicle in the same way. A stock solution of glibenclamide (Sigma, St. Louis, MO) was made by mixing $25 \mathrm{mg}$ in $10 \mathrm{~mL}$ dimethylsulfoxide (DMSO), and the injection/infusion solution was made by mixing $200 \mu \mathrm{L}$ stock in $2.3 \mathrm{~mL}$ unbuffered normal saline (NS; $0.9 \% \mathrm{NaCl}$ ) and clarifying the solution using a few microliters of $0.1 \mathrm{~N} \mathrm{NaOH}$ (final $\mathrm{pH} \sim 8.5$ ). The osmotic mini-pumps delivered $1.0 \mu \mathrm{L} / \mathrm{h}$, yielding infusion doses of $200 \mathrm{ng} / \mathrm{h}$. This treatment is estimated to yield a serum concentration of $<10 \mathrm{nM}$, and has minimal effects on serum glucose (Simard et al., 2006; Simard et al., 2007b; Simard et al., 2009b).

\section{Suppression of $A b c c 8$}

We used antisense oligodeoxynucleotide, 5'-GGCCGAGT GGTTCTCGGT-3' (Abcc8-AS), for in-vivo gene suppression (Yokoshiki et al., 1999). Scrambled oligodeoxynucleotide, 5'-TGCCTGAGGCGTGGCTGT-3' (Abcc8-Scr) was used as control, and both were phosphorothioated at four distal bonds to protect against endogenous nucleases. Within $10 \mathrm{~min}$ of contusion injury, a loading dose of $300 \mu \mathrm{g}$ in $300 \mu \mathrm{L}$ NS was given IV, and mini-osmotic pumps (Alzet 2001D, $8 \mu \mathrm{L} / \mathrm{h}$ ) with jugular vein catheters were implanted that delivered oligodeoxynucleotides in NS at a rate of $1 \mathrm{mg}$ per rat per $24 \mathrm{~h}$ (Gerzanich et al., 2009).

\section{Histology and immunohistochemistry}

After euthanasia, the rats were perfused with heparinized NS followed by $4 \%$ paraformaldehyde. Tissues were cryoprotected using 30\% w/v sucrose. For routine histology, $10-\mu \mathrm{m}$ cryosections were stained using Nissl stain.

Cryosections $(10 \mu \mathrm{m})$ were post-fixed with $4 \%$ formaldehyde and immunolabeled using standard techniques. After 
permeabilizing $(0.3 \%$ Triton $\mathrm{X}-100$ for $10 \mathrm{~min})$, the sections were blocked ( $2 \%$ donkey or $5 \%$ goat serum for $1 \mathrm{~h}$; Sigma D9663), then incubated with primary antibody directed against: SUR1 (1:200 SC-5789; Santa Cruz Biotechnology, Santa Cruz, CA); NeuN (1:100 Clone A60; Chemicon, Temecula, CA); vimentin (1:200 C43; Sigma, St. Louis, MO); laminin (1:200 FITC-conjugated; EY Laboratories, San Mateo, CA). Fluorescent-labeled species-appropriate secondary antibodies (Molecular Probes, Eugene, OR) were used for visualization. Omission of primary antibody and competition with antigenic peptides, when available, were used as negative controls. Fluorescent signals were visualized using epifluorescence microscopy (Nikon 90i; Nikon Instruments, Melville, NY).

Unbiased measurements of capillary length in the different treatment groups were obtained using specialized commercial software (NIS-Elements, Advanced Research v3.06; Nikon Instruments). Cryosections $(10 \mu \mathrm{m})$ were immunolabeled for vimentin or laminin as above, and regions of interest (ROI) in the penumbra adjacent to the necrotic void were identified. For each rat, three ROI, each $350 \times 450 \mu \mathrm{m}$, were processed. Individual "objects" within each ROI were defined as areas with contiguous specific labeling $3 \times$ above background. The maximum length of each object was determined for objects $>5 \mu \mathrm{m}$ in size. Based on the rod model, the length of the central axis of a thin rod is given by: $\mathrm{L}=\left[\mathrm{P}+\left(\mathrm{P}^{2}-16 \times \mathrm{A}\right)^{0.5}\right] / 4$, where $\mathrm{P}$ and $\mathrm{A}$ are the perimeter and the area of the object, respectively. Frequency count histograms of object lengths (bin width $5 \mu \mathrm{m}$ ) were constructed by combining data from three rats in the vehicle-treated and the glibenclamide-treated groups.

Immunoblot. Lysates of injured brain tissues were prepared by homogenizing in RIPA lysis buffer. Lysates were run on electrophoretic gels (NuPAGE ${ }^{\circledR} 4-12 \%$ Bis-Tris gels; Novex, Invitrogen, Carlsbad, CA). SUR1 was detected using antiSUR1 antibody (SC-5789; Santa Cruz Biotechnology). The membranes were stripped and re-blotted for $\beta$-actin (1:5000; Sigma), which was used as a loading control. Detection was carried out using the ECL system (Amersham Biosciences, Inc., Piscataway, NJ) with imaging (Fuji LAS-3000; Fujifilm USA, Inc., Valhalla, NY) and densitometry (Scion Image; Scion Corp., Frederick, MD).
In-situ hybridization. In-situ hybridization was performed as previously described (Simard et al., 2007b; Simard et al., 2009a). Fresh-frozen sections were post-fixed in 5\% formaldehyde for $5 \mathrm{~min}$. Digoxigenin-labeled probes (sense: $5^{\prime}$-GC CCGGGCACCCTGCTGGCTCTGTGTGTCCTTCCGCGCCT GGGCATCG- $3^{\prime}$ ) were designed and supplied by GeneDetect (Brandenton, FL), and hybridization was performed according to the manufacturer's protocol (www.genedetect.com/ protocols.htm).

Tissue blood. After euthanasia, the rats were perfused with heparinized saline to remove intravascular blood. A 10$\mathrm{mm}$ coronal slab of ipsilateral hemisphere encompassing the lesion was homogenized and processed using Drabkin's reagent (Simard et al., 2007b).

Neurobehavioral assessments. Behavioral measurements were performed by two blinded evaluators. Spontaneous behavior was assessed using quantified vertical exploration ("rearing") (Frey et al., 2009; Nikulina et al., 2004). The rats were placed in a freshly-cleaned acrylic glass cylinder $(20 \mathrm{~cm}$ diameter $\times 20 \mathrm{~cm}$ height), and spontaneous behavior was recorded using a digital video camcorder. Vertical exploration was quantified as the number of seconds spent with both front paws elevated above shoulder height during the first 3 min spent inside the chamber.

\section{Statistical analysis}

The Student's t-test or one-way ANOVA with Bonferroni comparisons was used, as appropriate. A difference between groups was deemed to be significant if $p<0.05$.

\section{Results}

\section{Progressive secondary hemorrhage:}

Spatiotemporal progression of contusion

The model of focal cortical contusion that we studied was characterized by PSH (i.e., spatiotemporal progression of the contusion) (Fig. 1). When examined shortly after injury, surface views of the brain showed multiple islands of hemorrhagic lesions separated by regions of more normal-appearing
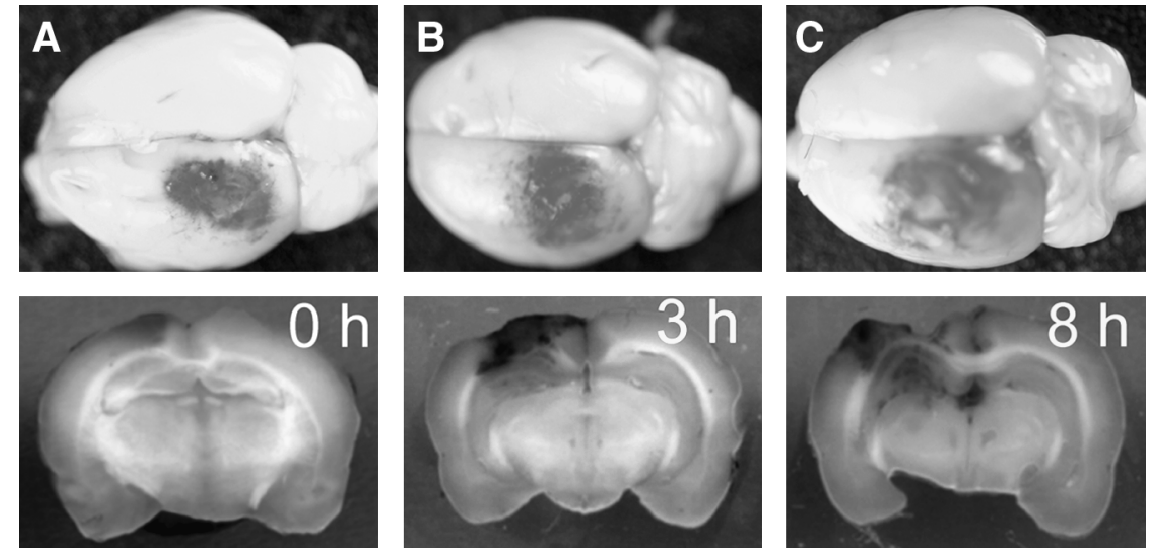

FIG. 1. Contusion injury results in progressive secondary hemorrhage. (A-C) Surface views (above), along with coronal views of the same brains (below), from rats euthanized immediately after, $3 \mathrm{~h}$ after, or $8 \mathrm{~h}$ after contusion injury, as indicated. All rats were perfused with saline to remove intravascular blood. The images shown are representative of three rats per group. 
tissue, typical of the appearance of a subpial hemorrhagic contusion and distinct from subdural and subarachnoid hemorrhage. The area involved was confined to the region of cortex struck by the impactor. Coronal views through the epicenters of the same brains showed that the hemorrhagic lesions at these early times were invariably confined to the
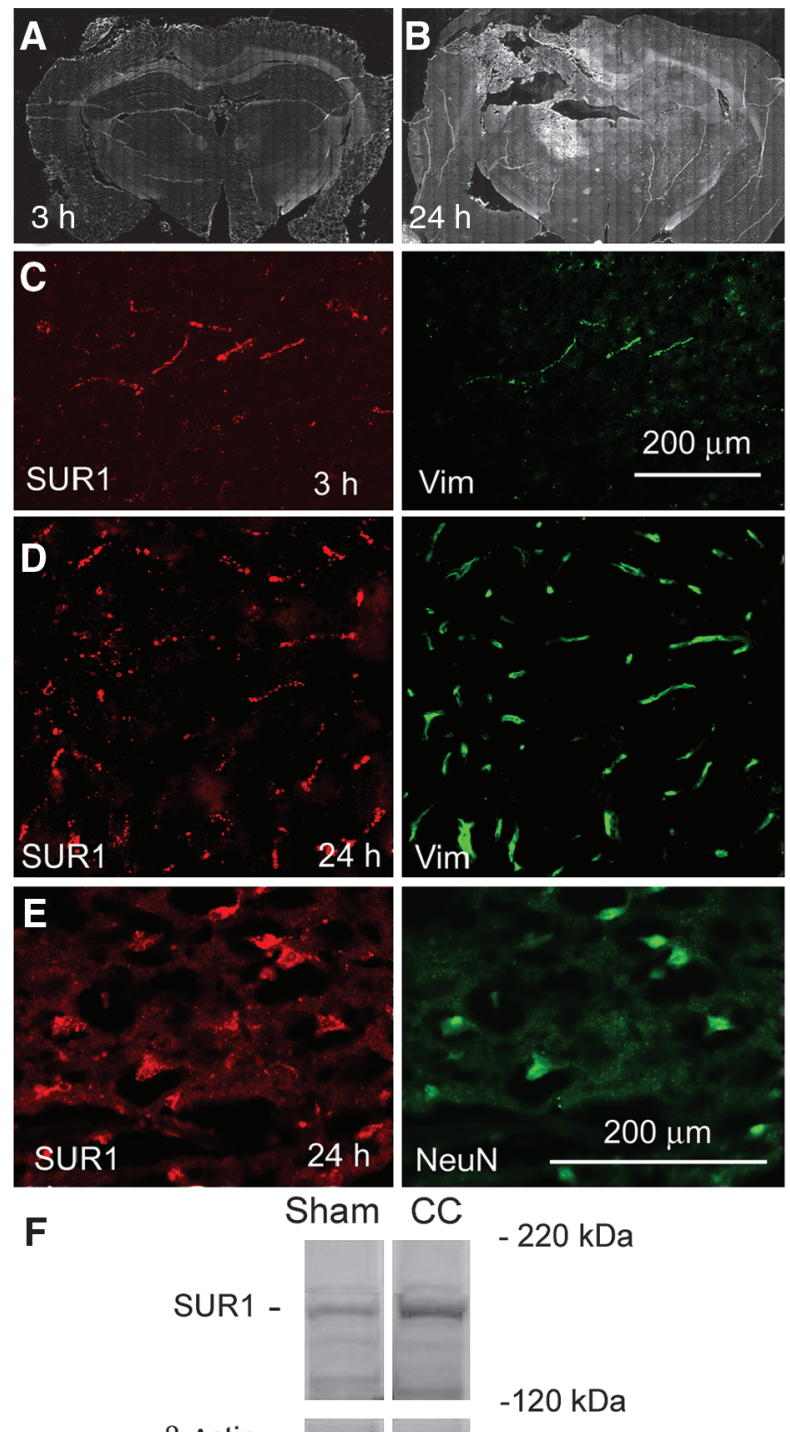

$-220 \mathrm{kDa}$

$\beta$-Actin -
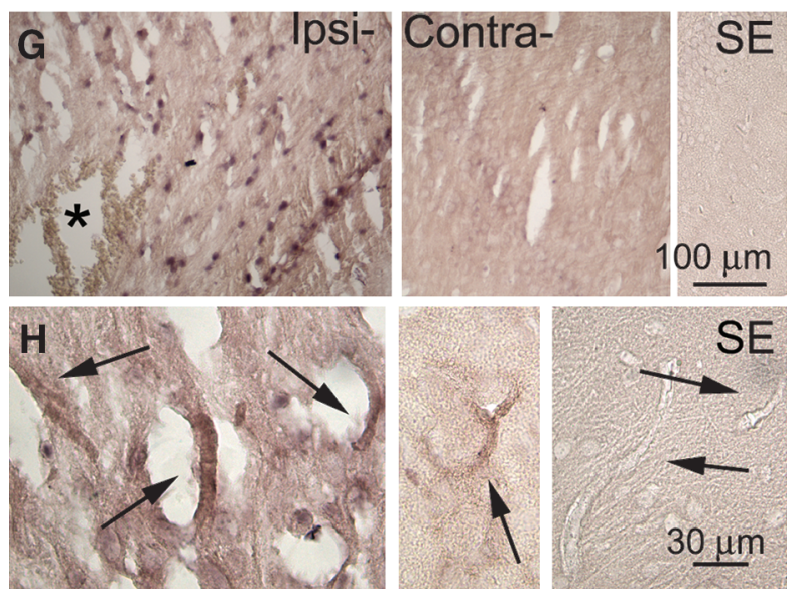

cortex, above the corpus callosum (Fig. 1A). At later times, surface views of the brain showed modest but noticeable expansion over the cortical surface in all directions, especially medially and laterally, as well as some filling in of the regions between islands that previously appeared devoid of hemorrhage. More striking were the coronal views of the same brains, which showed progressive spread of the hemorrhagic lesions to deeper tissues, including corpus callosum and hippocampus, by $3 \mathrm{~h}$ (Fig. 1B), and thalamus by $8 \mathrm{~h}$ (Fig. 1C), regions where hemorrhage was never apparent immediately after injury. Notably, the hemorrhagic contusion extending from cortex to underlying hippocampus at 3-8h resembled the distribution of $\operatorname{IgG}$ extravasated at the same times in a controlled cortical impact model (Hicks et al., 1997).

\section{Contusion traumatic brain injury and upregulation of sulfonylurea receptor 1}

The model of focal cortical contusion that we studied was associated with upregulation of SUR1 (Fig. 2). Immediately after injury, immunolabeled coronal sections through the epicenter showed a pattern of SUR1 expression that was the same as naïve or sham controls. However, by $3 \mathrm{~h}$ after injury, SUR1 expression became apparent in tissues beneath the impact, especially in capillaries (Fig. 2C and D). By $24 \mathrm{~h}$ after injury, SUR1 expression had become quite prominent, not only in the cortex but in the underlying structures deep to the cortical site of impact, including the hippocampus, thalamus, and beyond, and was evident in capillaries as well as neurons (Fig. 2B, D, and E). In some cases, SUR1 expression at 24h extended to the contralateral dorsomedial thalamus. The extent of SUR1 expression at $24 \mathrm{~h}$ mirrored the extent of the hemorrhagic contusion observed at the same time.

We used immunoblots and in-situ hybridization to confirm our findings on SUR1 immunolabeling, to exclude possible non-specific labeling due to tissue necrosis. Both immunoblots and in-situ hybridization confirmed that SUR1 was upregulated by contusion injury, and that penumbral capillaries were prominent among structures in which SUR1 was upregulated (Fig. 2F and G).

\section{Progressive secondary hemorrhage and glibenclamide}

To expand upon our observations of PSH detailed in Figure 1, we quantified the amount of extravasated blood present in

FIG. 2. Contusion injury results in progressive upregulation of sulfonylurea receptor 1 (SUR1). (A-E) Montages of images (A and $B$ ) or images at high magnification $(C-E)$ of coronal brain sections immunolabeled for SUR1 (white or red), obtained at $3 \mathrm{~h}(\mathrm{~A}$ and $\mathrm{C})$ and $24 \mathrm{~h}(\mathrm{~B}, \mathrm{D}$, and $\mathrm{E})$ postcontusion. Sections were co-labeled for vimentin (green) to identify capillaries (C and D), or for NeuN (green) to identify neurons (E). (F) Immunoblot for SUR1 of sham-operated (Sham), and of cortical contused (CC) brain $24 \mathrm{~h}$ post-injury. $\beta$-Actin is shown as a loading control. The data shown are representative of triplicate immunoblots. ( $\mathbf{G}$ and $\mathbf{H}$ ) In-situ hybridization of brain ipsilateral and contralateral to the contusion, as indicated, both labeled using antisense probe. Ipsilateral brain labeled with negative control sense (SE) probe is also shown (arrows point to capillaries; asterisk indicates petechial hemorrhage). The images shown are representative of triplicate labelings. 
contused tissues at various times after injury (Fig. 3). Two groups of rats were studied: a vehicle-treated control group and a group treated with glibenclamide, a potent selective inhibitor of SUR1. Within 10 min of injury, rats received an IP injection of vehicle or a loading dose of glibenclamide $(10 \mu \mathrm{g} / \mathrm{kg} \mathrm{IP})$, then they were implanted with a mini-osmotic pump for continuous delivery of vehicle or glibenclamide ( $200 \mathrm{ng} / \mathrm{h}$ ) subcutaneously. This dose of glibenclamide has repeatedly been shown to be too low to have a significant impact on serum glucose (Simard et al., 2006; Simard et al., 2007b; Simard et al., 2009b). At the designated time, intravascular blood was removed by post-mortem perfusion, and the remaining blood in tissue homogenates was quantified spectrophotometrically after converting to cyanomethemoglobin using Drabkin's reagent.

In vehicle-treated rats, the amount of extravasated blood increased progressively with time, with the total volume of blood doubling during the first $12 \mathrm{~h}$ after injury, after which it stabilized (Fig. 3A). Half of this increase occurred within $3-5 \mathrm{~h}$ of injury. The amount of extravasated blood present at $24 \mathrm{~h}$ was significantly greater than the amount present at $45 \mathrm{~min}$ (3.6 $\pm 0.5 \mu \mathrm{L}$ versus $8.4 \pm 0.5 \mu \mathrm{L} ; p<0.01$ ), consistent with PSH. By contrast, in glibenclamide-treated rats, there was very little increase in blood during the $24 \mathrm{~h}$ after injury, and the amount of extravasated blood present at $24 \mathrm{~h}$ was not significantly different than the amount present at $45 \mathrm{~min}$ ( $3.7 \pm 0.1 \mu \mathrm{L}$ versus $4.2 \pm 0.3 \mu \mathrm{L} ; p=1$ ). The marked reduction in hemorrhage with glibenclamide was confirmed by visual inspection of tissue homogenates (Fig. 3A, insert), and of unprocessed coronal sections (Fig. 3B and C), both obtained at $24 \mathrm{~h}$. These findings are consistent with elimination of PSH by block of SUR1 with glibenclamide.

\section{Progressive secondary hemorrhage and antisense against sulfonylurea receptor 1}

At the low dose used, glibenclamide is very selective for SUR1. Nevertheless, partial block of SUR2 could not be excluded, and at higher doses glibenclamide could potentially affect yet other ATP-binding cassette proteins (Golstein et al., 1999; Payen et al., 2001). To confirm specific involvement of SUR1 in PSH, we assessed the effect of antisense oligodeoxynucleotide directed against $A b c c 8$ ( $A b c c 8-A S)$, which is the gene that encodes SUR1. In addition, because transcriptional upregulation of SUR1 is known to be required for expression of functional SUR1-regulated $\mathrm{NC}_{\mathrm{Ca}-\mathrm{ATP}}$ channels (Simard et al., 2006), any effect of $A b c c 8$-AS on PSH would also suggest a requirement for de-novo transcription of SUR1. We
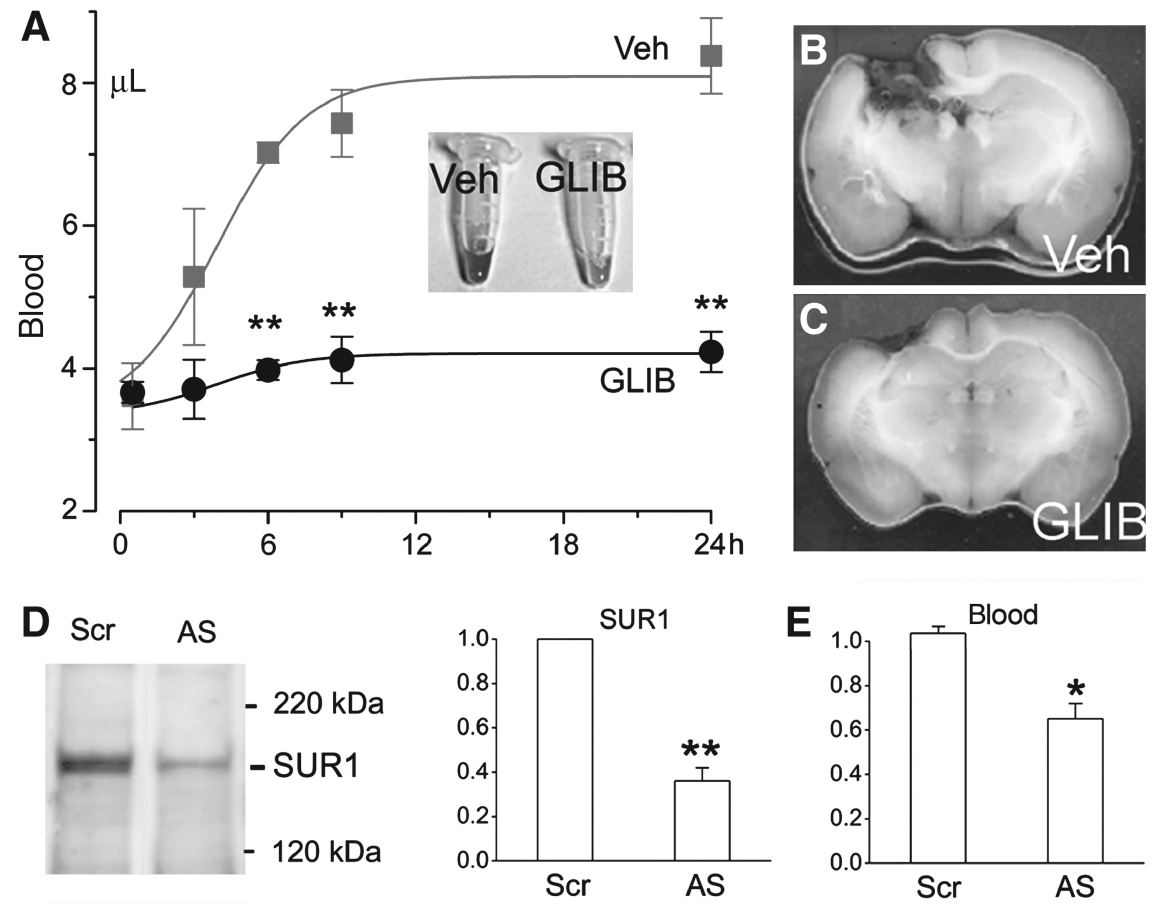

FIG. 3. Progressive secondary hemorrhage is blocked by glibenclamide and by antisense against $A b c c 8$. (A) The amount of extravasated blood present in contused brain at different times after injury in rats administered vehicle or glibenclamide $(10 \mu \mathrm{g} / \mathrm{kg}$ IP loading dose, followed by constant subcutaneous infusion at $200 \mathrm{ng} / \mathrm{h})$, starting within 10 min of injury. At the designated times, brains were perfused to remove intravascular blood, and extravasated blood in tissue homogenates was quantified spectrophotometrically after converting hemoglobin to cyanomethemoglobin using Drabkin's reagent. Data shown are mean \pm SE $\left({ }^{* *} p<0.01\right.$ by Bonferroni's comparison between vehicle-treated and glibenclamide-treated groups at the designated times; 3 rats per group at all but $24 \mathrm{~h} ; 5$ rats per group at $24 \mathrm{~h}$; test tubes containing homogenates at $24 \mathrm{~h}$ from rats administered vehicle or glibenclamide are also shown [insert]). (B and C) Images of coronal sections $24 \mathrm{~h}$ post-contusion from rats administered vehicle (Veh) or glibenclamide (GLIB). (D) Immunoblot for sulfonylurea receptor 1 (SUR1) of injured brains $24 \mathrm{~h}$ post-contusion from a rat administered $A b c c 8$-Scr (Scr) or Abcc8-AS (AS), as indicated. Densitometric analysis of immunoblots is also shown ( ${ }^{* *} p<0.01 ; 3$ rats per group). (E) Extravasated blood $24 \mathrm{~h}$ post-contusion, measured as above, in rats administered $A b c c 8-S c r$ or $A b c c 8-A S$, as indicated $\left({ }^{*} p<0.05\right)$. 
previously showed that antisense oligodeoxynucleotides are efficiently taken up by injured capillaries (Gerzanich et al., 2009).

Within 10 min of injury, rats were given a loading dose of Abcc8-AS followed by continuous IV infusion. Scrambled oligodeoxynucleotide (Abcc8-Scr) was used as control. Administration of $A b c c 8$-AS was associated with significant reduction of SUR1 expression in the same contusion model (Fig. 3D). Treatment with $A b c c 8$-AS also significantly reduced the volume of extravasated blood present at $24 \mathrm{~h}$, compared to Scr-Abcc8 (Fig. 3E), consistent with specific involvement of de-novo transcription of SUR1 in PSH.

\section{Capillary fragmentation and glibenclamide}

Persistent bleeding after trauma may be caused by a coagulopathy, thrombocytopathy, or vasculopathy. The beneficial effects of glibenclamide and of $A b c c 8$-AS on PSH could potentially have been mediated by promoting coagulation or platelet function, or by stabilizing the structure of fragile microvessels. However, glibenclamide was previously shown to have no effect on bleeding time in rats (Simard et al., 2007b), and to our knowledge oligodeoxynucleotides have not been reported to affect coagulation or platelet pathways. However, microvascular destruction has previously been reported after TBI (Lin et al., 2001; Park et al., 2009), raising the possibility that glibenclamide and Abcc8-AS might be acting to prevent the destruction of microvessels.

To examine the potential role of microvascular destruction in PSH, we studied two additional groups of rats: a vehicletreated control group and a group treated with glibenclamide, as described above. To assess microvascular structures postcontusion, we immunolabeled sections for laminin, a general marker of capillaries, and for vimentin, which is upregulated by activated endothelial cells (Frontczak-Baniewicz et al., 2007; Haseloff et al., 2006).

In vehicle-treated rats, penumbral structures that labeled positively for vimentin were foreshortened, irregular in shape, and seldom branched (Fig. 4A and C), which we interpreted to be consistent with capillary destruction and fragmentation. By contrast, in glibenclamide-treated rats, penumbral structures that labeled positively for vimentin were elongated and had the normal branching appearance of capillaries (Fig. 4B and C). Quantitative estimates of the lengths of vimentin-positive structures were obtained and used to construct histograms (Fig. 4C). Glibenclamide treatment was associated with significantly more structures $>50 \mu \mathrm{m}$ in length compared to controls. Precisely the same results were obtained with sections immunolabeled for laminin (Fig. 4D). These findings are consistent with the interpretation that PSH occurs because capillaries undergo fragmentation and loss of structural integrity in association with newly upregulated SUR1, and that block of SUR1 with glibenclamide or Abcc8-AS reduces PSH by preserving capillary integrity.

\section{Pathology}

We studied two additional groups of rats: a vehicle-treated control group and a glibenclamide-treated group, as described above, to determine histopathological involvement at $24 \mathrm{~h}$ (Fig. 5A-E). Coronal views through the epicenter of injury routinely showed that in both treatment groups, the cortex that had been subjected to contusion injury had been
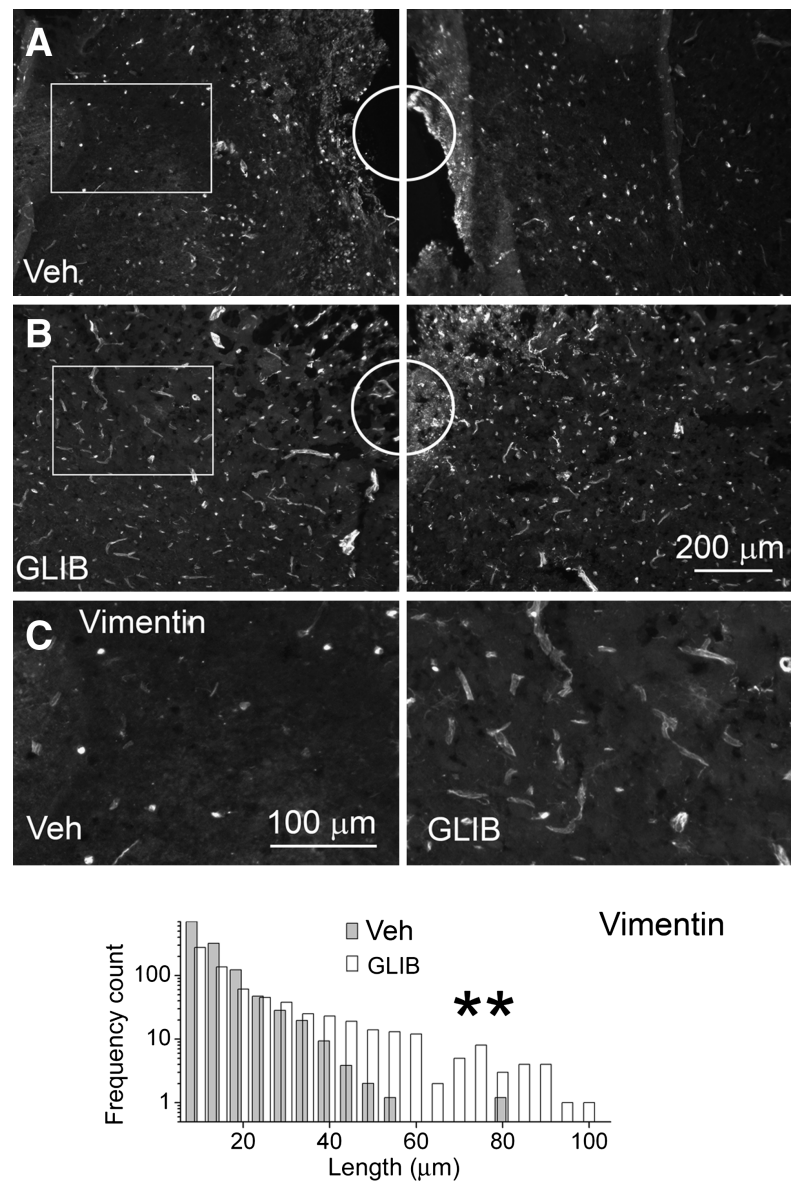

Vimentin
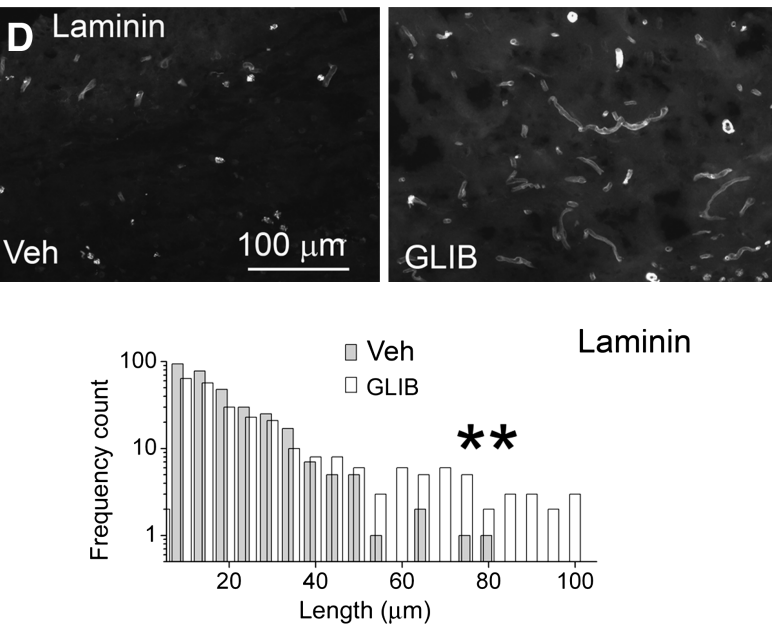

FIG. 4. Glibenclamide prevents capillary fragmentation after contusion. (A and B) Penumbral tissues medial and lateral to the contusion (denoted by the circle), immunolabeled for vimentin to show microvascular structures $24 \mathrm{~h}$ postcontusion in coronal brain sections from rats administered vehicle or glibenclamide, as indicated. (C) High-magnification images of rectangular areas outlined in A and B showing microvascular structures, and histograms showing the distribution of lengths of microvascular structures within the regions of interest, for rats administered vehicle or glibenclamide, as indicated. Significant differences in capillary lengths were evident for lengths $>50 \mu \mathrm{m}$ ( $\left.{ }^{* *} p<0.01\right)$. (D) Analysis of capillary length similar to that shown in C, using laminin for immunolabeling. 

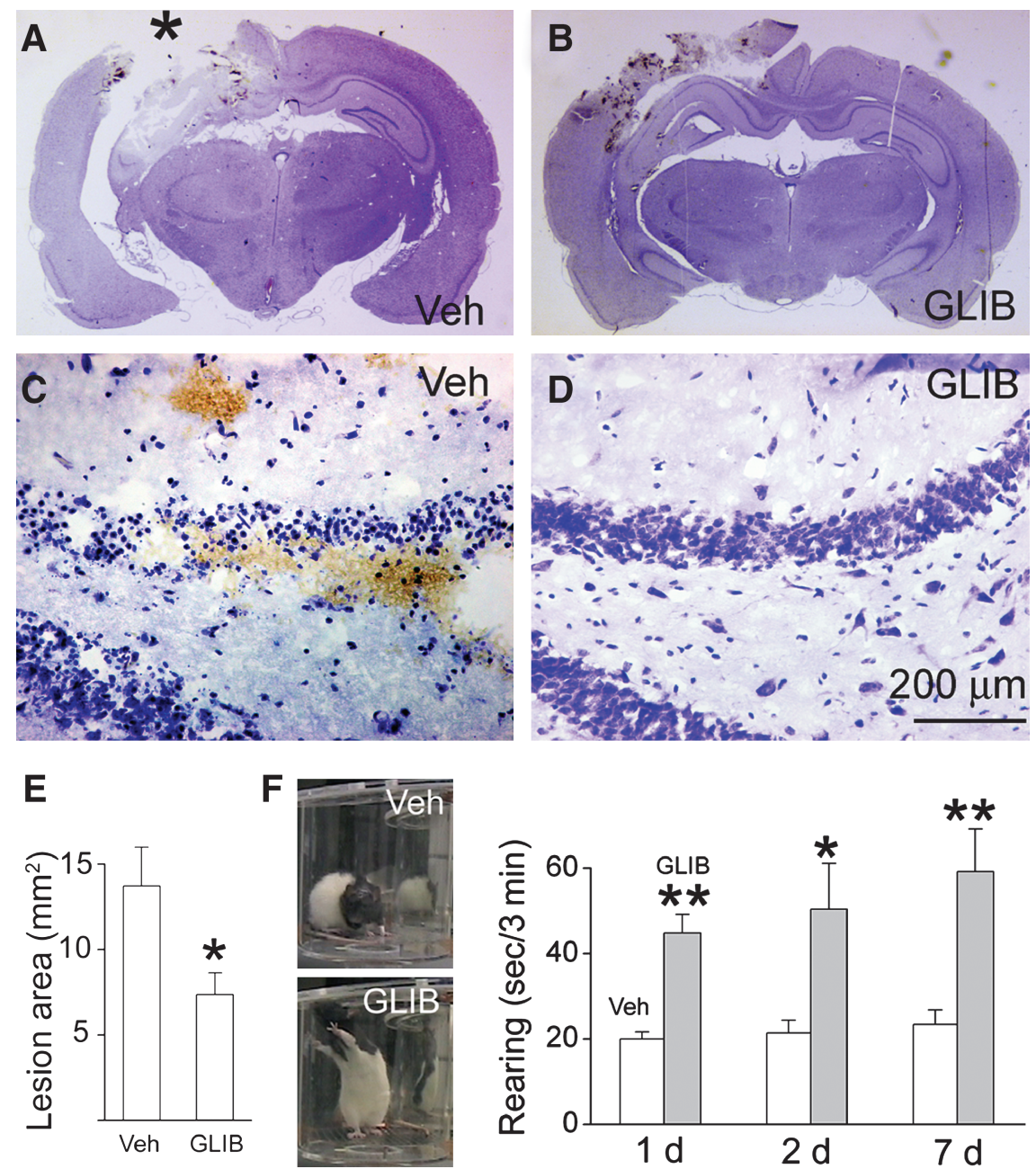

FIG. 5. Glibenclamide reduced lesion size and improved behavior after contusion. (A-D) Nissl-stained coronal sections of brains $24 \mathrm{~h}$ post-contusion, from four different rats administered either vehicle (Veh) or glibenclamide (GLIB), as indicated, with images shown at low magnification (A and B), or at high magnification, through the hippocampus (C and D). Note the petechial hemorrhages in C. (E) Lesion areas at $24 \mathrm{~h}$, measured from Nissl-stained sections through the contusion epicenter of rats administered vehicle (Veh) or glibenclamide (GLIB), as indicated (10 rats per group; $\left.{ }^{*} p<0.05\right)$. (F) Images of rats administered vehicle (Veh) or glibenclamide (GLIB), as indicated, inside of the cylinder used to quantify vertical exploration, and bar graph showing quantified vertical exploration (rearing) for the two groups of rats on the days indicated (7 rats per group; $\left.{ }^{*} p<0.05 ;{ }^{* *} p<0.01\right)$.

replaced by a necrotic void (Fig. 5A and B). However, in vehicle-treated rats the necrotic lesion extended deep to the cortex to involve the corpus callosum, hippocampus, and thalamus, whereas in glibenclamide-treated rats deeper structures, including the hippocampus and thalamus, were largely spared.

Examination of the hippocampi of vehicle-treated rats showed either total tissue destruction (Fig. 5A) or petechial hemorrhages and a paucity of neurons, with many of the remaining neurons appearing pyknotic (Fig. 5C). By contrast, hippocampal neurons in glibenclamide-treated rats were largely spared (Fig. 5B and D). These data not only corroborated previous observations that the extent of the hemorrhagic contusion early on predicts the eventual size of the necrotic lesion (Feeney et al., 1981; Sutton et al., 1993), but more importantly, showed the critical importance of PSH in determining the ultimate extent of tissue necrosis.
Lesion area at the epicenter was used to quantify the effect of the drug. Lesion areas in vehicle-treated rats were $~$ twofold larger than in glibenclamide-treated rats (Fig. 5E).

\section{Behavior}

In the final series of experiments, we sought to evaluate whether the short-term beneficial effects of glibenclamide on PSH observed within $24 \mathrm{~h}$ of injury would translate into later benefits in terms of behavioral outcomes. Again we studied two groups of rats: a vehicle-treated group and a glibenclamide-treated group, as described above, except that they were treated for 7 days post-contusion.

After TBI of various sorts, rats generally exhibit reduced spontaneous activity (Fromm et al., 2004; Vink et al., 2003). To quantify this behavioral abnormality, we measured time spent in spontaneous vertical exploration. On this measure, 
rats treated with glibenclamide fared significantly better than vehicle-treated rats, with the beneficial effect of drug being durable for the full week of observation (Fig. 5F).

\section{Discussion}

Primary traumatic brain injury is invariably complicated by secondary injury, which results in expansion of the original lesion and concomitant worsening of neurological outcome. Mechanisms of secondary injury are numerous and may include cytotoxic processes such as excitotoxicity, free radical damage, apoptosis, and inflammation. In addition, a major component of secondary injury results from microvascular dysfunction (Dietrich et al., 1994; Lin et al., 2001), including ischemia, edema, and spatiotemporal progression (blossoming) of hemorrhage, which we term PSH.

In humans, progressive hemorrhage is quite common, occurring in $25-50 \%$ of TBI cases, and is often associated with worse clinical outcomes (Chang et al., 2006; Oertel et al., 2002; Servadei et al., 1995; Smith et al., 2007). A variant of this condition may be the entity called "delayed traumatic intracerebral hemorrhage," in which hemorrhage is not evident initially, but develops a few hours later (Cooper, 1992; Gudeman et al., 1979; Mertol et al., 1991; Tseng, 1992; Young et al., 1984). Clinical experience indicates that the scattered small deep hemorrhages that are often seen in the context of diffuse axonal injury may be less prone to expansion, compared to frank subpial cortical contusions.

Post-injury hemorrhage has been observed in various animal models of TBI, including fluid percussion (Cortez et al., 1989; Dietrich et al., 1994; Dixon et al., 1987), controlled cortical impact (Dixon et al., 1991), and impact acceleration (Foda and Marmarou, 1994) models. To our knowledge, only rare reports of focal cortical contusion models have noted a progression of hemorrhage during the first few hours after injury (Feeney et al., 1981; Sutton et al., 1993), and no study has examined the early time course or the underlying cellular and molecular events responsible for progression. Small hemorrhages $\left(2-4 \mathrm{~mm}^{3}\right)$ at the anisotropic grey-white matter junction produced by lateral fluid percussion are reduced in size by various treatments (Atkins et al., 2007; Belayev et al., 2001; Belayev et al., 2002; Utagawa et al., 2008), but it is unclear whether the hemorrhagic lesions in untreated animals are larger because of a pathophysiological process similar to that seen in focal cortical contusion. Whether the scarcity of animal studies that address hemorrhagic progression is accounted for by the choice of models or by some other factor is not known. PSH is probably more likely and more apparent in models of focal cortical contusion, compared to models of diffuse injury such as impact acceleration and fluid percussion. Also, the specific parameters used to induce focal cortical contusion (shape of the impactor head, impact force, impact velocity, rate of deceleration, and depth of penetration) could potentially influence the likelihood as well as the pattern of hemorrhagic progression. Alternatively, a long-standing neurocentric orientation in TBI research may have resulted in limited interest in detailed examination of vascular events during the hyperacute first $12 \mathrm{~h}$ after injury.

In human $\mathrm{TBI}$, hemorrhagic progression has commonly been attributed to continued bleeding of microvessels fractured by the original trauma, or to a clinical or subclinical coagulopathy. However, an abnormality in the prothrombin time, partial thromboplastin time, or platelet count at admission is found in only half of these cases (Stein et al., 1992; Yadav et al., 2006). Moreover, a recent study of early administration of recombinant factor VII in patients with traumatic intracerebral hemorrhage has demonstrated only a modest attenuation of hematoma progression (Narayan et al., 2008). Such findings suggest that mechanisms unrelated to coagulation may contribute to hemorrhagic progression.

Here we propose for the first time the concept that expansion of a hemorrhagic lesion in the context of contusion injury may have as its basis a previously unrecognized mechanism of secondary injury with specific, well-defined molecular antecedents. After a focal traumatic insult that is sufficient to contuse brain tissue, the SUR1-regulated channel is transcriptionally upregulated in penumbral cells and microvessels within a few hours of injury. The traumatic event itself may induce transcriptional upregulation of the channel by way of a molecular mechanism yet to be uncovered. In addition, ischemia from mass effect, acting via hypoxiainducible factor 1, as well as the primary hemorrhage itself, acting via nuclear factor $-\kappa \mathrm{B}$, further augment transcriptional upregulation of the channel (Simard et al., 2006; Simard et al., 2009a). As long as energy is sufficient to maintain adequate levels of ATP, the channels remain closed and do no harm. However, critical depletion of ATP causes the channels to open, leading to persistent influx of sodium that is compounded by $\mathrm{Na}^{+}-\mathrm{K}^{+}$-ATPase pump failure, which is also due to ATP depletion. Sustained influx of sodium results in oncotic cell swelling (cytotoxic edema) and eventually oncotic cell death (Chen et al., 2001; Simard et al., 2006). This scenario may play out in neurons, astrocytes, and capillary endothelial cells, all of which have been shown to upregulate the channel. When endothelial cells are involved, oncotic cell swelling encroaches on the capillary lumen, compromising blood flow and compounding ischemia. Moreover, oncotic swelling of endothelial cells deranges the cytoskeleton, resulting in loss of integrity of interendothelial tight junctions and promoting the formation of vasogenic edema (Simard et al., 2009a). Ultimately, oncotic death of endothelial cells results in the complete loss of structural integrity of capillaries, yielding catastrophic failure that allows extravasation of blood to form petechial hemorrhages. With time, individual petechial hemorrhages increase in number and eventually coalesce into larger hemorrhagic lesions that encompass significantly more tissue than the original primary injury. This sequence of molecular and cellular events is corroborated by observations of microvascular failure in the region of injury, including progressive edema, progressive hemorrhage, progressive attenuation of regional cerebral blood flow, and progressive loss of microvessels (Engel et al., 2008; Park et al., 2009). Thus, PSH associated with TBI may be the end stage in a progressive process of microvascular failure, similar in nature to the progressive capillary dysfunction believed to occur with ischemia and hypoxia (Simard et al., 2007a).

The brain's response to trauma is temporally and spatially progressive (Bramlett and Dietrich, 2007; Povlishock and Jenkins, 1995). Numerous pathological processes evolve during the first several hours to days after trauma, including diffuse axonal injury, cell death, susceptibility to ischemia, and seizures, among others. Although brain contusion itself has been referred to as a "role model" for lesion progression (Kawamata and Katayama, 2007), the PSH that so often 
complicates cortical contusion has not generally been appreciated as reflecting the complex phenomenon of progressive microvascular failure described here. Implicit in the notion of progression is that underlying cellular and molecular mechanisms evolve over time. In the case of PSH, key events require several hours to transpire, including transcriptional upregulation of channel subunits and their assembly and transport to the cell membrane to form functional SUR1regulated $\mathrm{NC}_{\mathrm{Ca}-\mathrm{ATP}}$ channels. The fact that $\mathrm{PSH}$ requires several hours to play out bodes well for efforts aimed at aborting it by proper and timely treatment.

The microvascular dysfunction implicit with PSH is responsible not only for edema and ischemia, but the hemorrhage itself that characterizes $\mathrm{PSH}$ is exquisitely toxic to neural tissues due to free radical formation and inflammatory responses that are especially damaging to white matter (Regan and Guo, 1998; Wang et al., 2002). Acting together, these processes significantly amplify the primary injury, resulting in the autodestruction of brain tissue. These factors make PSH one of the most destructive mechanisms of secondary injury involving the CNS. The severity of the acute focal cortical contusion has previously been associated with the eventual size of the resulting necrotic lesion (Feeney et al., 1981; Sutton et al., 1993), but a role for PSH has not previously been suggested. Our data demonstrate a clear link between progressive hemorrhage in the hyperacute stage and eventual outcome days later, and that eliminating PSH during the first $12 \mathrm{~h}$ after injury was associated with a significant reduction in the size of the necrotic lesion, and a concomitant improvement in neurological function.

In rodent models of ischemic stroke, subarachnoid hemorrhage, and spinal cord injury, block of the SUR1-regulated $\mathrm{NC}_{\text {Ca-ATP }}$ channel by glibenclamide or other SUR1 inhibitors results in significant improvements in edema, lesion volume, neurological function, and mortality (Simard et al., 2006; Simard et al., 2007b; Simard et al., 2009a). In humans with diabetes mellitus, use of sulfonylureas before and during hospitalization for stroke is associated with significantly better stroke outcomes (Kunte et al., 2007). Results of the present study suggest that the SUR1-regulated $\mathrm{NC}_{\mathrm{Ca} \text {-ATP }}$ channel also plays a role in $\mathrm{PSH}$ following contusion injury to the brain, and that blockade of the channel with glibenclamide or preventing channel upregulation with antisense has significant beneficial effects. The possibility of administering antisense to reduce de-novo expression of SUR1-regulated $\mathrm{NC}_{\text {Ca-ATP }}$ channels, combined with glibenclamide to block channels already expressed, would appear to be a highly promising strategy for reducing secondary injury after focal cortical contusion.

\section{Acknowledgments}

This work was supported by grants to J.M.S. from the Department of Veterans Affairs (Baltimore, MD), the National Heart, Lung and Blood Institute (HL082517), the National Institute of Neurological Disorders and Stroke (NS061808 and NS060801), and the Christopher and Dana Reeve Foundation, and to V.G. from the National Institute of Neurological Disorders and Stroke (NS061934).

\section{Author Disclosure Statement}

JMS holds a US patent $(\# 7,285,574)$, “A novel non-selective cation channel in neural cells and methods for treating brain swelling." JMS is a member of the scientific advisory board of Remedy Pharmaceuticals and holds shares in the company. No support, direct or indirect, was provided to JMS, or for this project, by Remedy Pharmaceuticals. All other authors declare no conflicting financial interest.

\section{References}

Atkins, C.M., Oliva, A.A. Jr., Alonso, O.F., Pearse, D.D., Bramlett, H.M., and Dietrich, W.D. (2007). Modulation of the cAMP signaling pathway after traumatic brain injury. Exp. Neurol. 208, 145-158.

Belayev, L., Alonso, O.F., Liu, Y., Chappell, A.S., Zhao, W., Ginsberg, M.D., and Busto, R. (2001). Talampanel, a novel noncompetitive AMPA antagonist, is neuroprotective after traumatic brain injury in rats. J. Neurotrauma 18, 1031-1038.

Belayev, L., Becker, D.A., Alonso, O.F., Liu, Y., Busto, R., Ley, J.J., and Ginsberg, M.D. (2002). Stilbazulenyl nitrone, a novel azulenyl nitrone antioxidant: improved neurological deficit and reduced contusion size after traumatic brain injury in rats. J. Neurosurg. 96, 1077-1083.

Bramlett, H.M., and Dietrich, W.D. (2007). Progressive damage after brain and spinal cord injury: pathomechanisms and treatment strategies. Prog. Brain Res. 161, 125-141.

Buki, A., and Povlishock, J.T. (2006). All roads lead to disconnection?-Traumatic axonal injury revisited. Acta Neurochir. (Wien.) 148, 181-193.

Chang, E.F., Meeker, M., and Holland, M.C. (2006). Acute traumatic intraparenchymal hemorrhage: risk factors for progression in the early post-injury period. Neurosurgery 58, 647-656.

Chen, M., and Simard, J.M. (2001). Cell swelling and a nonselective cation channel regulated by internal $\mathrm{Ca} 2+$ and ATP in native reactive astrocytes from adult rat brain. J. Neurosci. 21, 6512-6521.

Chen, M., Dong, Y., and Simard, J.M. (2003). Functional coupling between sulfonylurea receptor type 1 and a nonselective cation channel in reactive astrocytes from adult rat brain. J. Neurosci. 23, 8568-8577.

Cooper, P.R. (1992). Delayed traumatic intracerebral hemorrhage. Neurosurg. Clin. N. Am. 3, 659-665.

Cortez, S.C., McIntosh, T.K., and Noble, L. J. (1989). Experimental fluid percussion brain injury: vascular disruption and neuronal and glial alterations. Brain Res. 482, 271-282.

Dietrich, W.D., Alonso, O., and Halley, M. (1994). Early microvascular and neuronal consequences of traumatic brain injury: a light and electron microscopic study in rats. J. Neurotrauma 11, 289-301.

Dixon, C.E., Clifton, G.L., Lighthall, J.W., Yaghmai, A.A., and Hayes, R.L. (1991). A controlled cortical impact model of traumatic brain injury in the rat. J. Neurosci. Methods 39, 253-262.

Dixon, C.E., Lyeth, B.G., Povlishock, J.T., Findling, R.L., Hamm, R.J., Marmarou, A., Young, H.F., and Hayes, R.L. (1987). A fluid percussion model of experimental brain injury in the rat. J. Neurosurg. 67, 110-119.

Engel, D.C., Mies, G., Terpolilli, N.A., Trabold, R., Loch, A., De Zeeuw, C.I., Weber, J.T., Maas, A.I., and Plesnila, N. (2008). Changes of cerebral blood flow during the secondary expansion of a cortical contusion assessed by 14C-iodoantipyrine autoradiography in mice using a non-invasive protocol. J. Neurotrauma 25, 739-753.

Feeney, D.M., Boyeson, M.G., Linn, R.T., Murray, H.M., and Dail, W.G. (1981). Responses to cortical injury: I. Methodology and local effects of contusions in the rat. Brain Res. 211, 67-77. 
Foda, M.A., and Marmarou, A. (1994). A new model of diffuse brain injury in rats. Part II: Morphological characterization. J. Neurosurg. 80, 301-313.

Frey, L.C., Hellier, J., Unkart, C., Lepkin, A., Howard, A., Hasebroock, K., Serkova, N., Liang, L., Patel, M., Soltesz, I., and Staley, K. (2009). A novel apparatus for lateral fluid percussion injury in the rat. J. Neurosci. Methods 177, 267-272.

Fromm, L., Heath, D.L., Vink, R., and Nimmo, A.J. (2004). Magnesium attenuates post-traumatic depression/anxiety following diffuse traumatic brain injury in rats. J. Am. Coll. Nutr. 23, 529S-533S.

Frontczak-Baniewicz, M., Walski, M., and Sulejczak, D. (2007). Diversity of immunophenotypes of endothelial cells participating in new vessel formation following surgical rat brain injury. J. Physiol Pharmacol. 58(Suppl. 5), 193-203.

Gerzanich, V., Woo, S.K., Vennekens, R., Tsymbalyuk, O., Ivanova, S., Ivanov, A., Geng, Z., Chen, Z., Nilius, B., Flockerzi, V., Freichel, M., and Simard, J.M. (2009). De novo expression of Trpm4 initiates secondary hemorrhage in spinal cord injury. Nat. Med. 15, 185-191.

Golstein, P.E., Boom, A., Jacobs, P., Masereel, B., and Beauwens, R. (1999). P-glycoprotein inhibition by glibenclamide and related compounds. Pflugers Arch. 437, 652-660.

Gudeman, S.K., Kishore, P.R., Miller, J.D., Girevendulis, A.K., Lipper, M.H., and Becker, D.P. (1979). The genesis and significance of delayed traumatic intracerebral hematoma. Neurosurgery 5, 309-313.

Haseloff, R.F., Krause, E., Bigl, M., Mikoteit, K., Stanimirovic, D., and Blasig, I.E. (2006). Differential protein expression in brain capillary endothelial cells induced by hypoxia and posthypoxic reoxygenation. Proteomics 6, 1803-1809.

Hicks, R.R., Baldwin, S.A., and Scheff, S.W. (1997). Serum extravasation and cytoskeletal alterations following traumatic brain injury in rats. Comparison of lateral fluid percussion and cortical impact models. Mol. Chem. Neuropathol. 32, 1-16.

Kawamata, T., and Katayama, Y. (2007). Cerebral contusion: a role model for lesion progression. Prog. Brain Res. 161, 235-241.

Kunte, H., Schmidt, S., Eliasziw, M., Del Zoppo, G.J., Simard, J.M., Masuhr, F., Weih, M., and Dirnagl, U. (2007). Sulfonylureas improve outcome in patients with type 2 diabetes and acute ischemic stroke. Stroke 38, 2526-2530.

Lin, B., Ginsberg, M.D., Zhao, W., Alonso, O.F., Belayev, L., and Busto, R. (2001). Quantitative analysis of microvascular alterations in traumatic brain injury by endothelial barrier antigen immunohistochemistry. J. Neurotrauma 18, 389-397.

Marmarou, A. (2007). A review of progress in understanding the pathophysiology and treatment of brain edema. Neurosurg. Focus. 22, E1.

Mertol, T., Guner, M., Acar, U., Atabay, H., and Kirisoglu, U. (1991). Delayed traumatic intracerebral hematoma. Br. J. Neurosurg. 5, 491-498.

Morales, D.M., Marklund, N., Lebold, D., Thompson, H.J., Pitkanen, A., Maxwell, W.L., Longhi, L., Laurer, H., Maegele, M. Neugebauer, E., Graham, D.I., Stocchetti, N., and McIntosh, T.K. (2005). Experimental models of traumatic brain injury: do we really need to build a better mousetrap? Neuroscience 136, 971-989.

Narayan, R.K., Maas, A.I., Marshall, L.F., Servadei, F., Skolnick, B.E., and Tillinger, M.N. (2008). Recombinant factor VIIA in traumatic intracerebral hemorrhage: results of a dose-escalation clinical trial. Neurosurgery 62, 776-786.

Nikulina, E., Tidwell, J.L., Dai, H.N., Bregman, B.S., and Filbin, M.T. (2004). The phosphodiesterase inhibitor rolipram delivered after a spinal cord lesion promotes axonal regeneration and functional recovery. Proc. Natl. Acad. Sci. U.S.A. 101, 8786-8790.

Oertel, M., Kelly, D.F., McArthur, D., Boscardin, W.J., Glenn, T.C., Lee, J.H., Gravori, T., Obukhov, D., McBride, D.Q., and Martin, N.A. (2002). Progressive hemorrhage after head trauma: predictors and consequences of the evolving injury. J. Neurosurg. 96, 109-116.

Park, E., Bell, J.D., Siddiq, I.P., and Baker, A.J. (2009). An analysis of regional microvascular loss and recovery following two grades of fluid percussion trauma: a role for hypoxia-inducible factors in traumatic brain injury. J. Cereb. Blood Flow Metab. $29,575-584$.

Payen, L., Delugin, L., Courtois, A., Trinquart, Y., Guillouzo, A., and Fardel, O. (2001). The sulphonylurea glibenclamide inhibits multidrug resistance protein (MRP1) activity in human lung cancer cells. Br. J. Pharmacol. 132, 778-784.

Povlishock, J.T., and Jenkins, L.W. (1995). Are the pathobiological changes evoked by traumatic brain injury immediate and irreversible? Brain Pathol. 5, 415-426.

Regan, R.F., and Guo, Y. (1998). Toxic effect of hemoglobin on spinal cord neurons in culture. J. Neurotrauma 15, 645653.

Servadei, F., Nanni, A., Nasi, M.T., Zappi, D., Vergoni, G., Giuliani, G., and Arista, A. (1995). Evolving brain lesions in the first 12 hours after head injury: analysis of 37 comatose patients. Neurosurgery 37, 899-906.

Simard, J.M., Chen, M., Tarasov, K.V., Bhatta, S., Ivanova, S., Melnitchenko, L., Tsymbalyuk, N., West, G.A., and Gerzanich, V. (2006). Newly expressed SUR1-regulated NC(Ca-ATP) channel mediates cerebral edema after ischemic stroke. Nat. Med. 12, 433-440.

Simard, J.M., Geng, Z., Woo, S.K., Ivanova, S., Tosun, C., Melnichenko, L., and Gerzanich, V. (2009a). Glibenclamide reduces inflammation, vasogenic edema, and caspase-3 activation after subarachnoid hemorrhage. J. Cereb. Blood Flow Metab. 29, 317-330.

Simard, J.M., Kent, T.A., Chen, M., Tarasov, K.V., and Gerzanich, V. (2007a). Brain oedema in focal ischaemia: molecular pathophysiology and theoretical implications. Lancet Neurol. $6,258-268$

Simard, J.M., Tsymbalyuk, O., Ivanov, A., Ivanova, S., Bhatta, S., Geng, Z., Woo, S.K., and Gerzanich, V. (2007b). Endothelial sulfonylurea receptor 1-regulated NC(Ca-ATP) channels mediate progressive hemorrhagic necrosis following spinal cord injury. J. Clin. Invest. 117, 2105-2113.

Simard, J.M., Woo, S.K., Bhatta, S., and Gerzanich, V. (2008). Drugs acting on SUR1 to treat CNS ischemia and trauma. Curr. Opin. Pharmacol. 8, 42-49.

Simard, J.M., Yurovsky, V., Tsymbalyuk, N., Melnichenko, L., Ivanova, S., and Gerzanich, V. (2009b). Protective effect of delayed treatment with low-dose glibenclamide in three models of ischemic stroke. Stroke 40, 604-609.

Smith, J.S., Chang, E.F., Rosenthal, G., Meeker, M., von Koch, C., Manley, G.T., and Holland, M.C. (2007). The role of early follow-up computed tomography imaging in the management of traumatic brain injury patients with intracranial hemorrhage. J. Trauma 63, 75-82.

Stein, S.C., Young, G.S., Talucci, R.C., Greenbaum, B.H., and Ross, S.E. (1992). Delayed brain injury after head trauma: significance of coagulopathy. Neurosurgery 30, 160-165.

Sutton, R.L., Lescaudron, L., and Stein, D.G. (1993). Unilateral cortical contusion injury in the rat: vascular disruption and temporal development of cortical necrosis. J. Neurotrauma 10, 135-149. 
Tseng, S.H. (1992). Delayed traumatic intracerebral hemorrhage: a study of prognostic factors. J. Formos. Med. Assoc. 91, 585589.

Utagawa, A., Bramlett, H.M., Daniels, L., Lotocki, G., Dekaban, G.A., Weaver, L.C., and Dietrich, W.D. (2008). Transient blockage of the CD11d/CD18 integrin reduces contusion volume and macrophage infiltration after traumatic brain injury in rats. Brain Res. 1207, 155-163.

Vink, R., O'Connor, C.A., Nimmo, A.J., and Heath, D.L. (2003). Magnesium attenuates persistent functional deficits following diffuse traumatic brain injury in rats. Neurosci. Lett. 336, 41-44.

Wang, X., Mori, T., Sumii, T., and Lo, E.H. (2002). Hemoglobininduced cytotoxicity in rat cerebral cortical neurons: caspase activation and oxidative stress. Stroke 33, 1882-1888.

Yadav, Y.R., Basoor, A., Jain, G., and Nelson, A. (2006). Expanding traumatic intracerebral contusion/hematoma. Neurol. India 54, 377-381.
Yokoshiki, H., Sunagawa, M., Seki, T., and Sperelakis, N. (1999). Antisense oligodeoxynucleotides of sulfonylurea receptors inhibit ATP-sensitive $\mathrm{K}+$ channels in cultured neonatal rat ventricular cells. Pflugers Arch. 437, 400-408.

Young, H.A., Gleave, J.R., Schmidek, H.H., and Gregory, S. (1984). Delayed traumatic intracerebral hematoma: report of 15 cases operatively treated. Neurosurgery 14, 22-25.

Address correspondence to: J. Marc Simard, Ph.D. Department of Neurosurgery 22 S. Greene Street, Suite S12D Baltimore, MD 21201-1595

E-mail: msimard@smail.umaryland.edu 
